\title{
Sobre la formación integral del médico: aportaciones de las Primeras Jornadas Estatales de estudio y reflexión sobre el tema
}

\author{
J.L. Tizón
}

\begin{abstract}
Se parte de las conclusiones de las Primeras Jornadas Estatales sobre la Formación Integral del Médico, que estaban planteadas no como unas jornadas de estudio sistemático, sino como unas jornadas de reflexión. En ese sentido, se incluyen en el artículo, tanto las conclusiones de los diversos grupos de trabajo de las jornadas, como una serie de reflexiones del propio autor sobre el tema, al menos en algunas de sus facetas fundamentales: influencia del contexto social, aspectos teóricos, técnicos y pragmáticos de las diversas propuestas para modificar la enseñaza de la medicina, su aplicación en la docencia pregrado, posgrado y continuada, selección de los docentes, de los discentes y de los métodos de evaluación. Se insiste en la necesidad de un cambio en la orientación del aprendizaje, introduciendo los elementos emocionales y experienciales en nuevos formatos técnicos, y en la utilidad y aplicaciones de los grupos en general y de los grupos 'tipo Balint' en particular en este nuevo contexto formativo.
\end{abstract}

Palabras clave. Aprendizaje. Clínica. Docencia médica. Enseñanza. Formación para la medicina. Grupos. Grupos 'Balint'. Sistemas docentes.

\section{On medical education: a reflection about the conclusions of the First State Conference on 'Doctor's integral education'}

Paper starts with the conclusions of the First State Conference about 'Doctor's Integral Education' that were outlined as a reflection conference more than a systematic study conference. In that sense, paper is based on the conclusions of the diverse Conference work groups. It includes a series of reflections on its topics: influences of the social context, theoretical aspects, technicians and pragmatic of the topic, their application in the pre-graduate teaching, graduate degree and continuous, selection of the docents, of the candidates and of the evaluation methods... We emphasize the necessity of a change in the orientation of the learning, introducing the emotional elements and experiential in new technical formats. We insist also in the utility and applications of the groups in general, and the 'Balint type' groups in particular, in this new formative context.

Key words. Balint groups. Clinic. Educational Systems. Formation for the medicine. Groups. Teaching. Teaching Medicine.

\section{Introducción: el contexto}

La medicina asistencial contemporánea en los países tecnológicamente desarrollados abunda en situaciones delicadas y al límite de la praxis clínica, que propician un encarecimiento de la asistencia, el desengaño y desgaste profesional $\mathrm{y}$ variados esoterismos alternativos. Para hacer más llamativa la situación, la población general, por un lado, critica los sesgos tecnoburocráticos de la medicina y a los médicos, pero, a la vez, aumenta su frecuentación, tanto en esa misma medicina que critica como en casi todo tipo de medicinas alternativas [1].

Hasta el extremo de que tal vez hoy ya sea lícito hacerse la pregunta de forma radical: ¿hay posibilidades de retomar y actualizar una perspectiva holística de la asistencia, la docencia y la investigación en medicina?

Algunos profesionales pensamos que sí, y de ahí nacieron las Jornadas, cuyas conclusiones glosamos en este trabajo. Para aceptar su orga-
Neuropsiquiatra del Institut Català de la Salut. Director del Equipo de Prevención en Salud Mental - Equipo de Atención Precoz a los Pacientes en Riesgo de Psicosis. Barcelona, España.

Correspondencia Equipo de Prevención en Salud Mental - Equipo de Atención Precoz a los

Pacientes en Riesgo de

Psicosis. Còrsega, 544.

E-08025 Barcelona.

E-mail

jtizon@gencat.cat

Agradecimientos En la gestación de este trabajo han sido fundamentales los intercambios mantenidos con los miembros del comité científico y organizativo, y con los ponentes de las Jornadas Estatales. En particular y casi con categoría de coautores, quiero citar expresamente a N. Daurella, E. Castell, X. Clèries, J. Clusa, V. Martí, A. Martín-Zurro, T. Morandi, A. Moretó, A. Vives, F. Borrell, P. Boschan, M. Broggi, R. Ciurana, R. Epstein, J. Guimón y R. Sender.

Las Jornadas Estatales de Estudio y Debate sobre el Futuro de la Formación Integral del Médico: ¿Bioingeniería o Medicina? fueron organizadas en Barcelona por el Institut d'Estudis de la Salut del Departament de Salut de 
Catalunya, la Fundació Congrés Català de Salut Mental, la Fundación CosmoCaixa, el Equipo de Prevención en Salud Mental-EAPPP del ICS, la CAMFIC, el Plan Director de Salud Mental y Adicciones y el Colegio de Médicos de Barcelona, el 8 y 9 de febrero de 2008 (www.fccsm.org) nización partimos de la comprobación de dos posiciones aparentemente contrapuestas entre los profesionales y los técnicos preocupados por este tipo de problemas de la docencia y la formación médicas, posturas que hace unos años esquematicé humorísticamente con los descriptivos de 'posición Bambi' o 'posición Godzilla' $[2,3]$. Para unos, en extremo, la clínica médica es tan sublime, delicada e inefable que no puede ni debe ser investigable, evaluable, sujeta a estudio de costes, sujeta a enseñanza y racionalizaciones tanto docentes como organizativas. La otra, hoy dominante, parecería afirmar algo así como 'déjate de tonterías y simplezas, lo importante es la eficiencia (económica), las cifras, los resultados (económicos estrechos), que las máquinas funcionen, que no haya problemas, y nada más...'

En ese sentido, uno de los grupos de trabajo de las Jornadas (coordinado por Pedro Boschan, José Guimón y Esperança Castell) caracterizó de la siguiente forma los elementos de ese contexto que habría que tener en cuenta para cualquier replanteamiento de la formación médica [1-12]:

- La humanización/deshumanización de la medicina se relaciona cada vez más directamente con el grado de humanización/deshumanización del contexto social en el que se practica.

- Las expectativas excesivas que una parte del sistema social deposita en la función del médico le colocan en una situación de gran exigencia, que dificultan la acogida y recepción adecuada de los pacientes. El profesional se defiende creando una distancia en la relación con el paciente debida en buena parte a los temores al desbordamiento y la confusión.

- Se ha avanzado con la creación de actividades de interconsulta y otros espacios de soporte a la atención primaria y la medicina interna por parte de los servicios especializados de salud mental, trabajo social, recursos humanos, etc. Pero esa aportación está limitada a pocas especialidades, y en ocasiones es difícil gestionar las incomprensiones que se generan en el trabajo conjunto [9]. A menudo, incluso se producen dinámicas cerradas de funcionamientos insatisfactorios que impiden la efectividad en la tarea. En este caso, los médicos pueden desmotivarse de una actitud más holística o integrada, incluso los médicos clínicos, y ello repercute tanto en la satisfacción de la po- blación como en la efectividad de la asistencia y la salud laboral de los propios médicos.

- Es notable entre los médicos y las organizaciones de docencia médica la falta de conocimientos y comprensión sobre el funcionamiento de los grupos y de las organizaciones, comenzando por el conflicto, omnipresente en cualquiera de ellos, entre realizar la tarea o diluir las responsabilidades, entre la responsabilidad pesonalizada y la colusión. Una repercusión sumamente extendida actualmente es la dilución de las responsabilidades de cada profesional, con la consecuente 'colusión en el anonimato' asistencial [2,18-20]. Otra repercusión consiste en la actitud, generalizada en las actuales administraciones, de no confiar en las capacidades de autogestión de los propios profesionales, hasta el extremo de que no se les suele permitir casi ninguna capacidad de gestión sobre las propias agendas (una situación que en algunas organizaciones hospitalarias se convierte en exactamente la contraria). Ante ello, no debemos olvidar que la gestión del tiempo que se dedica a cada paciente es, en último término, una cuestión básica en la formación del profesional para gestionar los propios recursos emocionales frente a los pacientes y frente a las exigencias del trabajo organizativo.

- Los médicos necesitan sentir que la administración confía en su profesionalidad, algo que hoy no sucede. Sin olvidar que, en ocasiones, son los propios profesionales quienes, sumidos en una actitud paranoide con respecto a la administración, dejan de aprovechar las ocasiones que la organización les ofrece para obtener un mayor rendimiento a los espacios de comunicación y poder recuperar el sentido de la tarea. Los directivos también necesitan tener sus propios espacios de reflexión sobre ello.

- A los profesionales y a las organizaciones no se les ayuda en la gestión de los límites y la culpa; más bien se tiende a favorecer la omnipotencia y la disociación: 'podemos con todo, y con lo que no podemos, no existe, no nos toca, no es nuestro trabajo o protocolo...' La incapacidad real de dar una atención de salud excelente, siempre y para todo el mundo, puede generar culpa en los profesionales. La gestión adecuada de la culpa supone tolerar limitaciones para realizar la tarea sin hacer una delegación sistemática de la responsabilidad hacia fuera $[1,20,21]$. 


\section{Modelos y teorías sobre la formación de los médicos}

Los miembros del comité organizador y el comité científico de las Jornadas (v. agradecimientos) pensamos que esas posturas extremas, si bien existen, no representan el sentir de numerosos profesionales y técnicos ni tampoco el futuro de la formación y la docencia en nuestra profesión; que deben existir otras vías y planteamientos, y que hoy se pueden renovar perspectivas y prácticas docentes e incluso investigar sobre las nuevas formas de ejercer y enseñar la medicina. Por eso se realizaron las Jornadas que glosamos como 'jornadas de trabajo e intercambio de experiencias', con una aportación organizativa importante, que hizo más compleja su organización. Todos los nuevos enfoques de la docencia $\mathrm{y}$ formación en medicina priman el valor de la experiencia personal y la experiencia grupal en dicha formación. Queríamos que esos sistemas formativos estuvieran ya incluidos en la organización de las Jornadas. Por esta razón, aunque no parece que 'estén los tiempos para la lírica', queríamos demostrarnos a nosotros mismos y a los asistentes que, con dificultades, necesidades de adaptación y problemas, las experiencias grupales pueden introducirse en todo tipo de sistemas docentes y formativos; mucho más hoy en día, cuando la formación para la entrevista, el trabajo en equipo y el trabajo en grupos se hallan tan empobrecidos en la medicina y en la enseñanza de la medicina habituales.

Como en todo tema técnico-científico, en el estudio de éste deberíamos atender al menos a los tres niveles que suelo proponer para aproximarse de forma holística a un tema científicotécnico: teórico, técnico y práctico [22-27].

En ese sentido, el primer nivel de enfoque del tema es el teórico: qué modelos y teorías existen hoy para la formación del médico. En ese ámbito podemos apreciar la existencia de un abanico de modelos que comienza con uno de formación para los médicos en el que teóricamente prima el biologismo; organizativamente, las facultades de medicina tradicionales, $y$ en el cual se emplean las técnicas piramidales, basadas en la asimetría y en la unidireccionalidad de la comunicación docente-discente $[24,25,27]$. En el otro extremo del abanico existen una serie de experiencias, propuestas y modelos, a menudo poco sostenidos, duraderos y apoyados. En términos genera- les, son propuestas o experiencias docentes que, de una forma u otra, intentan favorecer modelos teóricos integrales o biopsicosociales [26,27], con cambios notables en la organización de los sistemas de enseñanza y con técnicas docentes basadas en los grupos, en el crecimiento en grupos, en el intercambio de experiencias, en la relación personalizada tutor-discente, etc. En último lugar, se trata de la traslación a la docencia de la medicina de los dos modelos extremos con los cuales la medicina puede practicarse: el asimétrico-autoritario y el democrático, tendente a la simetría, basado en la comunicación bidireccional, en la experiencia relacional y en la autogestión emocional y organizativa [21,2,3,28-31].

En este sentido, no hay que olvidar que la formación y docencia se realizan con el fin de conseguir una competencia profesional, y la competencia médica incluye no sólo el uso de razonamientos clínicos y técnicas biomédicas, sino que hoy es definida como 'el uso habitual y juicioso de la comunicación, las herramientas y tecnologías sanitarias, el razonamiento clínico, las emociones, los valores y la autorreflexión en la práctica diaria para el beneficio de individuos y comunidades' [29]. Es decir, no es algo que se refiera tan sólo a conocimientos y/o técnicas biomédicas, sino a ese conjunto de factores. Además, no se adquiere puntualmente, ni con el final de carrera, ni con el MIR ni con el título de especialidad. La competencia se logra y se mantiene, sobre todo, gracias a la formación continuada, además de ser una cualidad contextual, es decir, referida a contextos sanitarios determinados, de tal forma que los profesionales con competencia acreditada en determinados contextos pueden resultar poco competentes en contextos sanitarios o médicos diferentes [25].

Las propuestas y experiencias que tienen en cuenta ese segundo tipo de orientaciones teóricas, técnicas y pragmáticas suelen tener en cuenta que ese cambio fue propuesto ya por Michael Balint en la década de 1950; más tarde, por los divulgadores del modelo biopsicosocial; luego, por los que defendemos la atención sanitaria centrada en el consultante como miembro de la comunidad (ASCCC) y por la línea de investigación y práctica de la Patient Centred Medicine y Patient Centred Communication, etc. [16,31-38].

Desde el punto de vista pragmático, para nuestra práctica clínica eso significa poder formarnos y desarrollar nuestras capacidades para la rela- 
ción médico-consultantes: empatía, solidaridad, capacidades de comunicación, capacidades de 'globalizar' nuestro conocimiento del paciente, orientación biopsicosocial de la práctica, etc.

Otro de los grupos de trabajo de las Jornadas (coordinado por Ronald Epstein, Jorge L. Tizón y Anna Moretó) se centró en el estudio de esos temas. En concreto, en definir 'qué y cómo debería enseñarse la medicina para conseguir esos profesionales capacitados para la atención en situaciones de complejidad de variables y de incertidumbre heurística, que son las habituales en la clínica. En ese sentido, seguramente hemos de partir del principio de que un clínico, un profesional asistencial y, en este caso, un profesional médico, no son científicos, ni deben ser fundamentalmente científicos, sino técnicos o tecnólogos que han alcanzado y pueden manejar una serie de competencias tanto a nivel de conocimientos teóricos como de técnicas, amén de las habilidades propias de sus saberes técnicos. Pero todo ello exige, además, un saber hacer en la práctica, ciertas capacidades y habilidades como artesano. Sin olvidar que, dada la complejidad de variables que se deben manejar en muchas de las especialidades y situaciones médicas, ello implica una capacidad para atender a la combinatoria de esos elementos adaptada a la situación concreta, a menudo con gran complejidad de variables, que es a lo que llamamos el 'arte del clínico'. Lo idóneo sería, pues, una formación que atendiera a esa realidad teórica y epistemológica de la medicina actual: una formación tecnológica, es decir, basada en técnicas apoyadas en conocimientos científicos, junto con una cierta capacidad de artesanía y de 'arte clínico' (Tabla I). Este técnico-artesano competente y adaptado al contexto, ese 'médico-profesional', queda bastante lejos del 'médico-sacerdote' y del 'médicocientífico', que aún se encuentran en según que facultades de medicina del mundo. Pero con esas otras propuestas, aún hoy bien extendidas, queda clara la importancia, muchas veces no declarada, de otros apartados de la formación médica: el mundo de las actitudes y de los valores. En último lugar, puede observarse cómo en esos diversos planteamientos aparecen abiertamente tres tipos de valores ideológicos que impregnan los principios de la enseñanza médica: los altruisticoreligiosos, los valores del cientificismo y los valores de la profesión como servicio público [4,5,6-8,31].

\section{Tabla I. Características de la competencia médica.}

Consiste en el uso habitual y juicioso de:

- La comunicación

- Técnicas

- Herramientas

- Razonamiento clínico

- Emociones

- Valores

- Autorreflexión

En la práctica diaria

Para el beneficio de individuos y comunidades

Es una combinación de:

- Conocimientos y razonamientos tecnológicos (orientados por la ciencia)

- Técnicas

- Artesanado y herramientas

- 'Arte' clínico

Otros dos grandes modelos, en este caso epistemológicos, se debaten hoy en el campo de la enseñanza de la medicina (y, prácticamente, de cualquier saber). Me refiero a la concepción del aprendizaje y, por lo tanto, a la formación, como proceso intelectual-racional frente a la formación y el aprendizaje como proceso más complejo, desde luego cognitivo, pero también emocional y experiencial. Es un debate que no debería existir si la formación del médico se apoyara en la ciencia, pues la ciencia psicológica ya hace decenios que ha demostrado la primacía del aprendizaje por experiencia y emocional. Su escasísima implantación en los estudios de medicina hace patente las enormes resistencias que se oponen al cambio de la formación médica.

Por todo ello, un objetivo para el cambio de modelos de enseñanza y para la ampliación de sus hoy estrechas miras estaría centrado en aprender a pensar también sobre lo emocional, mejorar la capacidad de observación e introspección emocional de los profesionales. Potenciar la práctica reflexiva, considerando que no es optativo ser reflexivo en la práctica profesional, sino que se trata de una obligación social básica para evitar enormes despilfarros y numerosos errores médicos $[31,33,36]$. 
Tabla Il. Algunos métodos de evaluación de la competencia médica.

Ejercicios escritos

- Cuestiones de elección múltiple

- Cuestiones clave y concordancia

- Preguntas cortas

- Ejercicios estructurados

Supervisiones

- Evaluaciones comentadas al final del período formativo

- Observación directa con checklist

- Exámenes orales

Simulaciones clínicas

- Pacientes simulados y exámenes clínicos estructurados por objetivos

- Pacientes estándares o simulados de incógnito

- Simulaciones de alta tecnología

Evaluaciones globales

- Evaluación por pares o compañeros

- Evaluación por los pacientes

- Autoevaluaciones (reevaluadas)

- Portafolios

Tutorías longitudinales
Tabla III. Métodos para evaluar la 'focalización en el consultante' con unidades de interacción teóricas, de tiempo o lingüísticas (adaptado de $[33,50])$.

Observación directa de las entrevistas seguida por:

- Sistemas de codificación

- Análisis interaccional

- Checklist

- Escalas de evaluación

Observación directa con pacientes simulados

Autoinformes del médico y el consultante con escalas para evaluar:

- Soporte a la autonomía

- Confianza

- Conocimiento global del paciente

- Satisfacción

Puntuación de vídeos por parte de los pacientes

Entrevistas semiestructuradas posconsulta

Grupos focales y grupos 'tipo Balint'

(evaluación por pares en grupo participante)

Observación etnoantropológica de campo

Evaluación por pares, por colegas o compañeros

Portafolios y evaluaciones 'multifocales'

sible durante la formación. Los conocimientos, habilidades y actitudes psicológicas y psicosociales dependende 'formas de ser,' 'humanismo', 'humanizar la carrera o la práctica', etc. Es decir, no se trata de una enseñanza de técnicas, ni debe ser una enseñanza técnica, orientada por modelos tecnológicos [13-15,25].

Un modelo alternativo de formación médica, por el contrario, debe partir de la idea de que los elementos psicológicos, psicosociales y socioantropológicos que necesita el médico contemporáneo para su práctica son ámbitos científicos y técnicos pertenecientes a disciplinas del saber diferenciadas de la biología. Son conocimientos y habilidades que dependen de la psicología, la sociología, la antropología, la ética, la política, etc., y que para enseñar y aprender ese tipo de conocimientos también se necesitan sistemas 


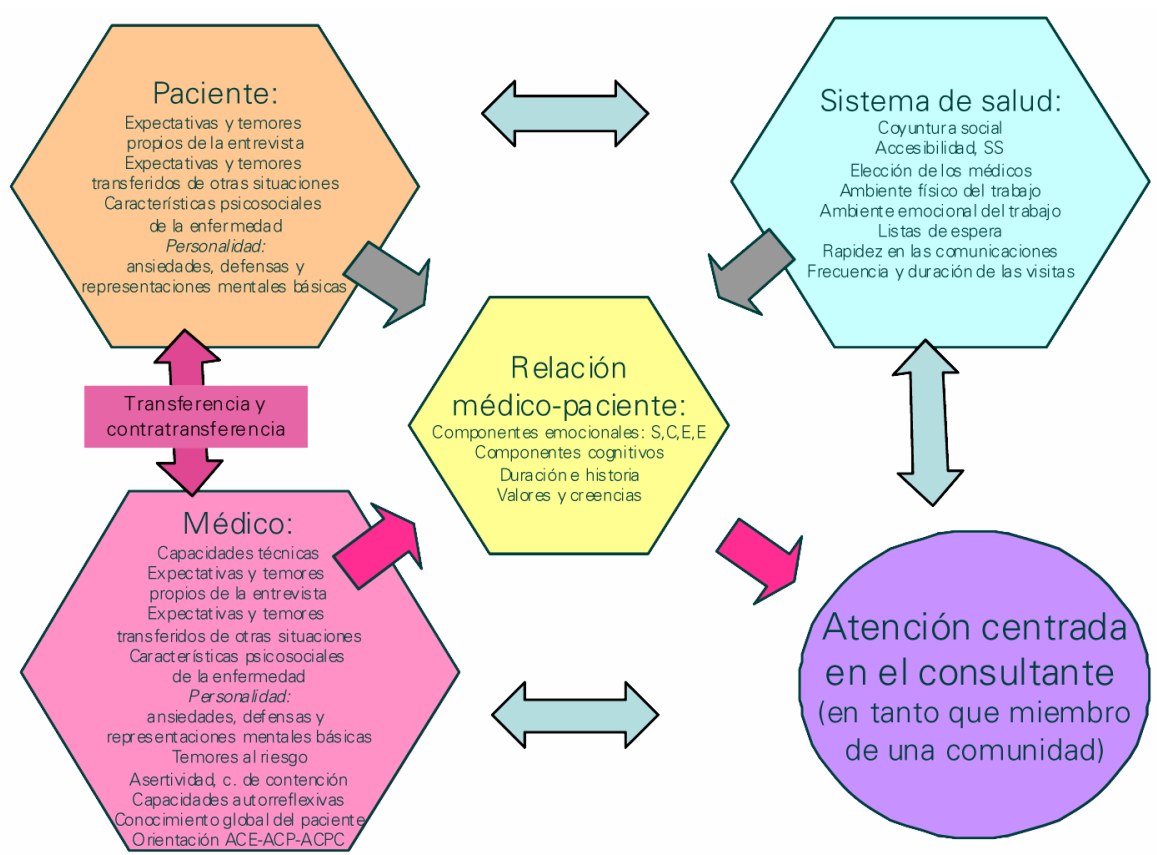

Figura. Relación médico-paciente: una perspectiva globalizadora (adaptada de [31]).

técnicos precisos, que en algunos casos hace más de un siglo que existen.

Desde un punto de vista estructural o sistémico, por otra parte, no hay que olvidar que según cuáles sean los sistemas de evaluación, su práctica a medio plazo sesga de forma notable tanto la enseñanza como la preparación de los alumnos. En nuestro país el tema se ha hecho patente al observar el sesgo que los sistemas de evaluación de los exámenes del MIR han impuesto sobre la formación, actividades e incluso sobre la vida personal de los estudiantes de los últimos cursos de carrera: primacía de lo 'teórico'-memorístico, primacía del apartado de 'acumulación de conocimientos' con una atención a determinadas habilidades muy parcial y ocasional (para la entrevista, para la exploración, para la relación asistencial, para el trabajo en equipo, etc.); atención casi inexistente a las actitudes necesarias para ser un clínico competente en habilidades psicosociales; restricción de la vida personal y de los intercambios y experiencias durante los últimos años de carrera, etc. Y esto a pesar de que hoy disponemos de sistemas y técnicas de evaluación numerosos, bien diferentes de los tradicionales, y muchos de ellos ampliamente estudiados desde el punto de vista empírico [29] (Tablas II y III). Porque, si bien no está 'arquitrabado' totalmente un modelo alternativo, hoy sí sabemos que, por ejemplo, las técnicas y evaluaciones deben ser múltiples para que se adapten a las distintas necesidades personales y contextuales.

\section{Sobre la selección del profesorado, las técnicas docentes y la evaluación}

Como es de esperar, eso supone inmediatamente comenzar a pensar en quién enseña y qué formación hay que requerir al docente, es decir, en la formación y preparación de los docentes [29,33,37,38].

El grupo de trabajo dedicado al tema cuestionó ampliamente los sistemas de selección y evaluación actuales del profesorado, que tienden a valorar tan sólo el aspecto investigador: publicaciones e investigaciones realizadas. Esto suele conllevar un auténtico desprecio por la práctica clínica, por la experiencia clínica, incluso por las 
capacidades e historia personal de innovaciones y organizaciones clínicas y asistenciales, que deberían ser, junto con las capacidades docentes y sus técnicas específicas, los elementos determinantes de la elección de formadores de médicos clínicos. Sin embargo, probablemente esos docentes y tutores de médicos 'formados para la ciencia' deberían coexistir en las facultades con los profesores y tutores formados fundamentalmente para la clínica, aunque el papel principal en la docencia les debería corresponder a estos segundos [39]. El médico clínico se forma, ante todo, pudiendo hacer suyas las habilidades, las actitudes y los algoritmos clínicos de su tutor o tutores, de ahí la importancia de disponer de unos buenos clínicos como tutores.

Otro gran defecto del tipo de formación actual es que no tiene en cuenta ni potencia las aportaciones grupales a la formación, ni siquiera la de los grupos de pares, la de los compañeros de grupo y formación, algo que hoy se sabe que es de fundamental importancia para el resultado de una formación clínica -tanto, que incluso ha dado lugar a numerosas obras de arte, gráficas o narrativas (cuadros, novelas, filmes sobre el tema). Sin embargo, la formación actual tiene muy poco en cuenta la utilidad de los grupos de observación, los grupos de reflexión y las videograbaciones, que permiten observarnos durante la acción. Y, desde luego, ni siquiera se plantean las aportaciones de los consultantes y sus familias a esa formación, aportaciones que se podrían facilitar por los modernos medios de sonido e imagen y los registros videográficos computarizados y sincronizados del discente y los consultantes. En el camino, a menudo se pierde la utilización seria y organizada de actividades docentes tales como las supervisiones, las tutorías, los grupos de reflexión sobre el material clínico, el análisis grupal de entrevistas grabadas, la utilidad de las técnicas de role playing, la utilidad formativa de los grupos de reflexión sobre las experiencias de enfermedad familiares o propias y sobre los procesos de duelo y pérdida -algo consustancial a la práctica de la medicina clínica [40-45].

Sin olvidar que, en este ámbito, no parece que sean necesarios cambios excesivamente complicados de realizar o técnicas muy complejas. Tan sólo se trata de abrir la puerta a la posibilidad de reflexionar y no sólo actuar, de abrir la puerta a técnicas que ayuden a los clínicos a realizar aproximaciones más parsimoniosas, cuando sea posible, y a experiencias docentes para aprender a tolerar la confusión -un componente fundamental de la práctica clínica real- y las 'ansiedades persecutorias' - un componente frecuente y, hoy en día, tal vez aumentado. Todo ello debería poderse integrar en un modelo formativo que estimulara la autorreflexión, la mindfulness $[12,27,33,44,45]$, que mejorara nuestras capacidades para pensar antes de actuar, saber reconocer la propia fatiga y el estado de ánimo, saber cuándo avanzar en la exploración, en la anamnesis, en nuestra intensidad de trabajo, y cuándo detenerse. Un modelo que incluso debería proporcionar indicadores de cuándo el propio clínico debe pedir ayuda, cuándo debe consultar por sus problemas personales vinculados con el trabajo [36], entre otros.

Otro elemento clave que hemos mencionado más arriba es la evaluación de la formación, cómo se evalúa el desarrollo formativo y docente de los alumnos. El sesgo de toda la formación de los médicos en la España actual provocado por algo que inicialmente ha sido un importante avance en la misma, los exámenes MIR, es hoy, sin embargo, sumamente visible. Además, sabemos que los sistemas de evaluación influyen siempre, a medio o corto plazo, en los contenidos y modelos docentes, que será un elemento a tener en cuenta en la modificación de la formación durante el pregrado, el MIR y las subespecialidades [46-51].

En este sentido, hay que apreciar avances como las ACOE: en términos generales, han supuesto un instrumento útil para cambiar el sistema de enseñanza/aprendizaje. Pero, en la medida en la que los estudiantes conocen los elementos evaluables, esta técnica puede facilitar un nuevo sesgo en la enseñanza de la medicina clínica, en el sentido de facilitar la incorporación de entrevistas y procesos interpersonales rutinarios, artificiales o artificiosos, hasta convertirlos en una caricatura de la entrevista. Para evitarlo, se ha propuesto, por ejemplo, cambiar el listado de evaluación posterior, investigar sobre otros apartados, practicar preguntas más abiertas, etc. Pero, posiblemente, es un tema en el que la última palabra la tendrían los tutores experimentados y clínicamente orientados, por un lado, los compañeros, por otro, y los propios consultantes, si fuéramos capaces de 'arquitrabar' sistemas de formación y evaluación en los cuales pudieran participar los tres colectivos con suficiente obje- 
tividad, organización y coherencia. Inicialmente, los grupos 'Balint' y 'tipo Balint' significaron un intento de introducir en el cambio docente tanto las emociones y reflexiones promovidas por los propios consultantes o pacientes, como las emociones y reflexiones promovidas por los pares, por los compañeros del grupo y por el grupo como organismo docente.

En general, en el terreno de la evaluación, la serie de recursos técnicos hoy disponible es muy amplia, como ya hemos visto (Tablas II y III) $[29,33,50]$. Actualmente se están intentado introducir nuevas perspectivas en la evaluación de la competencia de las profesionales, una evaluación desde ángulos diferentes, con el desideratum de las así llamadas 'evaluaciones desde los 360 grados': Si queremos evaluar el éxito de nuestros esfuerzos en formación biopsicosocial necesitamos no sólo la evaluación de los profesores y tutores, la del propio discente, la de los consultantes y también la de los colegas. En particular, es relativamente sencillo incorporar la evaluación por pares, pues muchas de nuestras debilidades y capacidades como clínicos las conocen mejor nuestros compañeros que nuestros profesores o tutores. Los pares pueden evaluar con mayor libertad los hábitos de trabajo, las actitudes, las habilidades personales, la interacción con los colegas y con los pacientes. Ronald M. Epstein, de la Universidad de Rochester, ponente en las Jornadas, presentó numerosos trabajos de investigación empírica sobre esas otras perspectivas y ámbitos para la evaluación, la mayoría de ellos con resultados muy positivos $[29,16,33,50,51]$.

\section{Aplicación de las aportaciones anteriores en los diversos momentos de la formación integral del médico}

Fue el tema en el que se centraron las reflexiones del segundo grupo de trabajo de las Jornadas, coordinado por A. Martín-Zurro y Xavier Clèries.

En la formación pregrado posiblemente es necesario hoy un cambio de perspectiva. Los objetivos deberían centrarse en la consecución de un profesional médico básico, fundamentalmente asistencial y competencialmente preparado para iniciar un período de formación especializada.

Si se tienen en cuenta esos objetivos, el proceso enseñanza-aprendizaje pregraduado debería asumir las siguientes características básicas de la docencia: formación teórica y práctica más compensada, con mayor importancia de la formación para la práctica y para la asistencia; integración de los contenidos básicos y clínicos mayor; tutorización activa y continuada del alumno y formación en el seno de grupos pequeños; contacto precoz con las organizaciones y dispositivos asistenciales; espacios de formación común interprofesionales; exposición más compartida con los paradigmas del ámbito biológico y con los psicológicos y psicosociales propios de la relación asistencial. Todo esto implica el desarrollo de un currículo con una estructura troncal inicial seguida de distintas líneas de formación diferenciadas.

Por lo que hace a los contenidos, una formación pregraduada actualizada debería contemplar de forma prioritaria cambios y ampliaciones en los siguientes ámbitos: comunicación y relación asistencial, con atención especial a las capacidades de observación, reflexiva y de autoconocimiento (mindfulness); atención primaria de salud; promoción de la salud y prevención de la enfermedad; bioética, antropología y sociología, y una información más amplia y menos sesgada de las llamadas medicinas complementarias, es decir, unos contenidos y una forma de asimilarlos orientada por el modelo de atención centrado en el consultante como miembro de la comunidad (ASCCC) $[31,42,43]$ (Tabla I).

La evaluación de la formación pregraduada debería garantizar, a través de distintos instrumentos y métodos, esa suficiencia competencial más equilibrada en términos de conocimientos, habilidades y actitudes del médico básico. Por ello, es importante la introducción de métodos de evaluación que potencien la bidireccionalidad, la detección de necesidades de aprendizaje y la introducción de medidas de reorientación docente que permitan su abordaje.

En la formación posgrado, hay que partir de la importancia del sistema MIR y avanzar hacia una estructuración troncal de la formación, imprescindible para conseguir la transversalidad competencial de los médicos formados: una estructuración con troncos básicos que faciliten una elección posterior más fundamentada de la especialidad y la posibilidad de migrar entre especialidades y subespecialidades.

El tutor, su formación, acreditación y reconocimiento son elementos nucleares del sistema docente en la especialización. La evaluación, en 
especial en este período, habría que organizarla como parte del proceso de formación, con elementos de carácter sumativo y formativo, para garantizar la suficiencia competencial del médico formado. La evaluación global del médico al finalizar su período formativo no debería consistir en una prueba o examen único, sino en el conjunto de toda la información evaluativa acumulada durante la formación y complementarla, cuando sea preciso, con otros elementos objetivos de comprobación.

En cuanto a la formación continuada, los replanteamientos anteriores harían pensar en que habría que situarla, conceptual y operativamente, en el contexto más amplio del Desarrollo Profesional Continuo individualizado (DPCI). Las estrategias tipo DPCI deberían utilizarse no sólo como forma de mejorar y controlar la formación continuada de nuestros clínicos, sino que pueden contribuir de forma decisiva a combatir el burnout profesional. Sin embargo, habría que introducir mecanismos de garantía de la calidad, eficacia, eficiencia y efectividad sobre la práctica obtenidos por las formas y actividades formativas diversas y, en general, del DPCI. Entre otros motivos, porque la financiación de las actividades por la industria farmacéutica, una tendencia hoy en auge en los tres niveles de la formación, puede introducir sesgos de deformación, de aún mayor tendencia a la medicalización y conflictos éticos en las intervenciones de los profesionales. A pesar de todo, parece que la recertificación periódica de los profesionales debe ser una apuesta decidida, condicionada a una accesibilidad suficiente a las actividades del DPCI, y basándola en métodos objetivos dirigidos a la ayuda y apoyo de los profesionales, más que a su control.

\section{Grupos 'Balint' y grupos de reflexión 'tipo Balint'}

Como decíamos al principio, uno de los orígenes iniciales de la organización de estas Jornadas era reflexionar acerca de la aportación de los grupos 'Balint' en la formación integral del médico, en especial hoy en día, cuando existen experiencias y estudios sobre su aplicación en los tres momentos de la formación de los médicos: pregrado, especialización y formación continuada [1820,31,43,57-64].
Recordemos que los grupos 'Balint' y similares toman ese nombre del psicoanalista de adopción británica Michael Balint, que ya desde mediados del siglo xx comenzó a practicarlos en el Reino Unido. Hoy podemos describirlos como grupos de reflexión en los cuales los médicos participantes, ayudados por un psicoanalista experto en grupos, reflexionan en común sobre sus dificultades de relación con un paciente o un tipo de pacientes determinado, con el fin de mejorar y ampliar sus capacidades para la relación asistencial [18-20,32,54-56].

En este sentido, el grupo de trabajo de las Jornadas dedicado al tema (coordinado por Neri Daurella, Ramón Ciurana e Ignacio López), así como los grupos de sensibilización 'tipo Balint' realizados durante las propias Jornadas (coordinados por Teresa Morandi y Àngels Vives), aportaron una serie de reflexiones que aquí podríamos resumir como sigue: las personas que conocen directamente el tema mediante prácticas docentes, clínicas o de investigación se reafirman en el valor tanto docente como de 'ayuda psicológica a los clínicos' que estos grupos, cuando son bien llevados, pueden jugar para con las inquietudes y problemas cotidianos y de formación psicosocial de los médicos clínicos.

En ese sentido, aunque el contexto en el que se empezaron a desarrollar estos grupos es muy diferente al actual, parece que aún son una herramienta importante en la formación, por las razones expuestas en otros lugares [18-20,32,54-56] y que fueron revisadas a lo largo de las Jornadas. Por ejemplo, es evidente que el fomento de la función reflexiva, de la que hablábamos anteriormente, y que hoy se enfoca también desde otras perspectivas y paradigmas psicológicos [29,33-36,44,45], se trata de una necesidad formativa prioritaria de los profesionales de salud actuales. Los grupos 'Balint' (GB) y de reflexión son un espacio idóneo en el cual ejercitar dicha función. Los problemas de la relación médico-paciente, de las relaciones entre profesionales de la salud o de la relación de éstos con los entornos de los pacientes siguen vigentes, y probablemente han adquirido una dimensión más compleja que cuando se crearon los GB. Además, el profesional sanitario afronta presiones crecientes que le desvirtúan de la realidad del acto médico o que añaden nuevas emociones y sentimientos a la ya cargada 'agenda diaria' del clínico: los GB son un instrumento útil para la contención de esas ansiedades y sufrimientos y 
para la corrección de algunos desenfoques que progresivamente se amplían si no se ponen 'remedios descontaminantes' a tiempo.

En general, el grado de satisfacción de los médicos que realizan la experiencia de participar al menos durante un año en un GB es alto [32,65-67]. Los profesionales suelen valorar especialmente que el GB les ayuda a interesarse por sus pacientes, a hacer más comprensibles los problemas y a sentirse ellos mismos menos frustrados. Sin embargo, hay que asumir que se dan casos en los que la experiencia del GB ha sido negativa, por deficiencias en su planteamiento o manejo (p. ej., cuando el uso inadecuado de la interpretación por parte del coordinador ha generado rechazo en el profesional, o cuando el coordinador no ha sabido crear y mantener un clima contenedor y no persecutorio en el grupo). En ese sentido, para el desarrollo de esa técnica docente, el grupo de trabajo sobre el tema consideraba que era fundamental la formación y capacidades grupales del coordinador para dirigir el grupo, de manera que pueda crear un clima motivador y de confianza desde la primera sesión. El coordinador también debe establecer muy claramente, desde el primer momento, las reglas de juego: qué se puede esperar del GB y qué no (expectativas realistas). Hoy se tiende a revitalizar las orientaciones y normas técnicas del propio Michel Balint y su laboratorio inicial, cuando aconsejaba explorar previamente las motivaciones para iniciar la experiencia y los posibles candidatos que debían participar. Aunque, en otros aspectos, los grupos que hoy se realizan difieren ampliamente de los realizados por el propio Balint y sus colaboradores directos [31,32,65-67]; por ejemplo, tienden a ser grupos multidisciplinares, que incluyen tipos de profesionales diversos y médicos con unnivel de formación diferente. En el caso de los residentes, tras discutir el dilema obligatoriedad-voluntariedad, algunas experiencias de larga duración en nuestro país $[54,55]$ parecen recomendar una inmersión en el GB mínima de tres meses, seguida de una experiencia optativa de uno o más años.

En este terreno, como en otros muchos de las innovaciones docentes necesarias, tanto los que intervinieron en las Jornadas como los propios grupos de trabajo o reflexión insistieron en la necesidad de plantear cambios, técnicas y procedimientos en la formación integral del médico que sean coherentes, sobre los que se posean suficien- te experiencia y pruebas empíricas, y que sean aplicados de forma honesta: no puede admitirse que se practiquen cambios docentes realizados por supuestos especialistas cuya competencia, formación y experiencia en la especialización no se conoce, como ha pasado con cierta frecuencia en la utilización de grupos y experiencias grupales, incluso de grupos 'tipo Balint'.

En definitiva, hemos de llegar a entender la formación del médico, como todo proceso formativo, en tanto que un conjunto emocional y cognitivo. En este sentido, ha de asentarse en unas emociones de base, promover unos conocimientos, habilidades y actitudes, y aprovechar para ello el clima emocional y grupal cotidiano [68]. Y en ese camino, la honestidad y coherencia de los formadores siguen siendo valores y variables de radical importancia para el resultado.

\section{Cinco conceptos básicos}

\section{Parcialización de la asistencia}

Una tendencia pragmática y técnica que evoluciona en los últimos decenios dentro de la medicina tecnológica: el desarrollo importante de todo tipo de técnicas, tanto exploratorias como terapéuticas, conlleva la dificultad de que puedan ser dominadas por un sólo profesional. Esa realidad, junto con la pluripatología o comorbilidad en muchos consultantes, dificulta ya de entrada una visión integral, holística o biopsicosocial del paciente. La propia ideología biologista y mecanicista de la medicina dominante facilita la dedicación parcial de cada especialista al 'hígado', el 'corazón' o 'las plaquetas' del consultante.

\section{Integralidad de la asistencia}

El intento de que alguna instancia del proceso sanitario (médicos, clínico de referencia, centro de salud, etc.) logre integrar los diversos aspectos biológicos de la asistencia y éstos con los psicológicos y los sociales.

\section{La formación como \\ proceso cognitivo-emocional}

Normalmente se piensa en la formación, la docencia y la discencia como algo fundamental- 
mente cognitivo, intelectual. Desde el psicoanálisis hasta los estudios psicológicos cognitivos modernos o de la psicología experimental, todos los datos apuntan a que el aprendizaje es un proceso mixto, cognitivo-emocional, y que si ambos elementos se utilizan organizadamente en la docencia mejoran los resultados.

\section{La formación como proceso de interacción grupal}

Lo mismo puede decirse del aprendizaje como logro individual: cada vez hay más datos, y más incontrovertidos, sobre el importante papel que los grupos y las experiencias grupales desempeñan en el aprendizaje $y$, en particular, en los aprendizajes a medio y largo plazo.

\section{Atención sanitaria centrada en el consultante como miembro de una comunidad}

Un submodelo de la perspectiva biopsicosocial que hace hincapié en los rendimientos de centrarse en las necesidades, experiencia y presentación del individuo que acude a la medicina, en la necesidad de no convertir a cada consultante en paciente y en la necesidad de tener en cuenta en la asistencia sanitaria los valores, sistemas grupales, ideologías y organizaciones de la comunidad.

\section{Bibliografía}

1. Ciurana R, Comín E, Marquet R, Martín A. Eppur si muove: los avances de la medicina y su difusión en el último decenio. FMC 2004; 11: 535-9.

2. Tizón JL. Los nuevos modelos asistenciales en medicina: una visión a partir de algunos de sus fundamentos psicológicos y epistemológicos. Revisiones en Salud Pública 1995; 4: 57-83.

3. Tizón JL. A propósito del modelo biopsicosocial, 28 años después: epistemología, política, emociones y contratransferencia. Aten Primaria 2007; 39: 93-7.

4. Broggi M. Gestión de valores 'ocultos' en la relación clínica. Med Clin (Barc) 2003; 121: 705-7.

5. Broggi M. El principio (en la relación clínica). Jano 2004; 1502: 42-3.
6. Gracia D. Humanismo médico y teología. Jano 2006; 6 de octubre de 2006. p. 1621.

7. Gracia D. Humanismo médico y positivismo. Jano 2006; 27 de octubre de 2006. p. 1624.

8. Gracia D. Contribución de las humanidades médicas a la formación del médico. Revista de la Fundación Medicina y Humanidades Médicas. URL: http://www.fundacionmhm.org/www_humanitas_es_numerol/revista.html. [30.06.2008].

9. Sender R, Daurella N, Martín-Zurro A, Borrell F. La realidad de la formación psicológica y psicosocial del médico en nuestro país. Ponencia presentada en las Primeras Jornadas sobre la Formación Integral del Médico: ¿Bioingeniería o Medicina? Barcelona: IES; 2008 (en preparación)

10. López-Piñero JM. Hacia una ciencia sociomédica: las ciencias sociales en la enseñanza médica. Med Clin (Barc) 1971; 65: 13-22.

11. Laín-Entralgo P. Vida, muerte y resurrección de la historia de la medicina. In Albarracín A, López-Piñero JM Granjel LS, eds. Medicina e historia. Madrid: Universidad Complutense; 1980. p. 22.

12. Borrell F, Epstein RM. Preventing errors in clinical practice: a call for self-awareness. Ann Fam Med 2004; 2: 310-6.

13. Tizón JL. Epistemology, politics, emotions and counter transference: around 'The biopsychosocial model 25 years later: principles, practice and scientific inquiry’. Annals of Family Medicine electronic letter de 17 de marzo de 2005 en la discusión del trabajo Borrell-Carrio F, Suchman AL, Epstein R. The biopsychosocial model 25 years later: principles, practice and scientific inquiry. Ann Fam Med 2004; 2: 576-82.

14. Tizón JL. El modelo biopsicosocial: la práctica de la teoría es imposible sin una política para la práctica. Respuesta del autor. Aten Primaria 2007; 39: 451-3.

15. Tizón JL. Humanismo y medicina. Jano, Medicina y Humanidades 2008; 1679: 25-31.

16. Epstein R, Borrell F, Tizón JL. Modelos y teorías en las que se basa la formación médica: ¿Existe en la realidad el modelo biopsicosocial? Ponencia presentada en las Primeras Jornadas sobre la Formación Integral del Médico ¿Bioingeniería o Medicina? Barcelona: IES; 2008 (en preparación).

17. Sender R, Salamero M. Programa de atención psicológica para los alumnos de la Facultad de Medicina de la Universidad de Barcelona. Barcelona: Universidad de Barcelona 2006. p. 5-11.

18. Balint M. El médico, el paciente y la enfermedad. Buenos Aires: Libros Básicos; 1969

19. Balint E, Norell JS, eds. Seis minutos para el paciente. Buenos Aires: Paidós; 1979.

20. Daurella N. La aportación de los grupos Balint: una perspectiva actualizada. Ponencia presentada en las Primeras 
Jornadas sobre la Formación Integral del Médico: ¿Bioingeniería o Medicina? Barcelona: IES; 2008 (en preparación).

21. Daurella N. Algunas aportaciones del psicoanálisis a la práctica de la medicina. Revista de Psicoterapia 2005; 62: 53-68.

22. Bunge M. Treatise on basic philosophy. Vol. VII. Philosophy of science and technology. Part II: Life Science, Social Science and Technology. Dordrecht: Reidel; 1985.

23. Chalmers AF. ¿Qué es esa cosa llamada ciencia? Madrid: Siglo XXI; 1987.

24. Tizón JL. Introducción a la epistemología de la psicopatología y la psiquiatría. Barcelona: Ariel; 1978.

25. Tizón JL. El futuro de la formación integral del médico: ¿bioingeniería o medicina? Jano, Medicina y Humanidades 2008; 1678: 52-4.

26. Engel G. The need for a new medical model: a challenge for biomedicine. Science 1977; 196: 129-36.

27. Borrell-Carrio F, Suchman AL, Epstein R. The biopsychosocial model 25 years later: principles, practice and scientific inquiry. Ann Fam Med 2004; 2: 576-82.

28. Clèries $\mathrm{X}$. La comunicación: una competencia esencial para los profesionales de la salud. Barcelona: ElsevierMasson; 2006.

29. Epstein RM. Assessment in medical education. N Engl J Med 2007; 356: 387-96.

30. Borrell F. La entrevista clínica. Barcelona: semFYC; 2007.

31. Tizón JL. Componentes psicológicos de la práctica médica. 6 ed. Barcelona: Biblària; 2000.

32. Tizón JL. Sobre los grupos Balint, el movimiento Balint y el cuidado de la relación médico-paciente. Aten Primaria 2005; 36: 453-5.

33. Epstein RM, Street RL. Patient centered communication in cancer-care: promoting healing and reducing suffering. Bethesda: National Cancer Institute, NIH; 2007.

34. Stewart M, Brown JB, Weston WW, McWhinney IR, MCWilliam CL, Freeman TR. Patient-centered medicine: transforming the clinical method. London: Sage; 1995.

35. Morse JM, Jonhson JL, eds. The illness experience: dimensions of suffering. New York: Sage; 1991.

36. Tizón JL. ¿Profesionales 'quemados', profesionales 'desengañados' o profesionales con trastornos psicopatológicos? Atención Primaria 2004; 33: 326-30.

37. Boschan P. La enseñanza de la salud mental en medicina: ¿formación o trasformación? Ponencia presentada en las Primeras Jornadas sobre la Formación Integral del Médico: ¿Bioingeniería o Medicina? Barcelona: IES; 2008 (en preparación).

38. Borrell F, Epstein RM, Pardell H. Profesionalidad y profesionalismo: fundamentos, contenidos, praxis y docencia. Med Clin (Barc) 2006; 127: 337-42.

39. De Groot JM, Kennedy SH. Integrating clinical and research psychiatry. J Psychiatr Neurosci 1995; 20: 150-4.

40. Woof WR, Carter YH. The grieving adult and the general practitioner: a literature review in two parts. Br Gen Pract 1997; 47: 443-8 y 509-14.

41. Woof WR. The future of bereavement care in British general practice. Eur J Cancer Care 1997; 6: 133-6.

42. Tizón JL. Pérdida, pena, duelo: vivencias, investigación y asistencia. Barcelona: Paidós; 2004.

43. Tizón JL. El humor en la relación asistencial. Barcelona: Herder; 2005.

44. Fitzgerald FT. On being a doctor. Curiosity. Ann Intern Med 1999; 130: 70-2.

45. Holmes J, Neighbour R, Tarrier N, Hinselwood RD, Bolsover $\mathrm{N}$. All you need is cognitive behaviour therapy? BMJ 2002; 324: 288-94.

46. Martín-Zurro A. Evaluations of postgraduate training and professional certification and re-certification of family doctors in various countries (United Kingdom, United States, Canada, Holland, Australia and Spain). Aten Primaria 2002; 30: 46-56.

47. Martín-Zurro A, Lafuente OA, Solsona M, Treviño R, Merino M, Ferrer L. Health professional need trends in Catalonia. Demographic study of Catalonia health professions. Cah Sociol Demogr Med 2006; 46: 407-527.

48. Martín-Zurro A. Objectives and methods of assessment of professional competence. Around Miller's pyramid. Med Clin (Barc) 2006; 127: 293-4.

49. Martín-Zurro A. Editorial: formación y necesidades de médicos. FMC 2008; 15: 2005-6.

50. Epstein RM, Franks P, Fiscella K, Shields CG, Meldrum SC, Kravits RL, et al. Measuring patient-centered communication in patient-physician consultations: theoretical and practical issues. Soc Sci Med 2005; 61: 1516-28.

51. McDaniel SH, Beckman HB, Morse DS, Silberman, J Seaburn DB, Epstein RM. Physician self-disclosure in a primary care visits: enough about you, how about me? Arch Intern Med 2007; 167: 1321-6.

52. Guimón P, Boschán P, Epstein R. Alternativas para una formación integral del médico: ¿se trata sólo de mejorar las habilidades de comunicación? Ponencia presentada en las Primeras Jornadas sobre la Formación Integral del Médico: ¿Bioingeniería o Medicina? Barcelona: IES; 2008 (en preparación).

53. Morandi T, Castelló E. La relación médico-paciente y los grupos Balint. Aten Primaria 1884; 1: 309-12.

54. Tizón JL. Los 'grupos de reflexión' en la atención primaria de Salud. I. Su origen. Atención Primaria 1993; 6: 309-14.

55. Tizón JL. Los 'grupos de reflexión' en la atención primaria de salud. II. Algunos elementos teóricos y técnicos. Aten Primaria 1993; 7: 361-7.

56. Tizón JL. Dai gruppi Balint ai gruppi di riflessione sulle componenti relazionali del servizio sanitario. Rivista di Psicoanalisi 1997; 63: 431-60.

57. Guimón J. Crise d'identidé et formation des psychiatres. 
Schweizer Archiv für Neurologie und Psychiatrie 1995; 146: 137-41.

58. Guimón J. Consequences of recent changes in research and practice for the identity of psychiatrists. Eur Psychiatry $1997 ; 12$ : 92-8.

59. Guimón J. New forms of psychiatric education: the Geneva experience. Eur J Psychiatry 2000; 14: 52-60.

60. Guimón J. Introduction aux therapies de groupe: théories, technique et programmes. Paris: Masson; 2001.

61. Guimón J. Training general practitioners in the psychoanalytical aspects of mental health. In Guimón J, Zac S, eds. Challenges of psychoanalysis in the 21st century. New York: Kluwer Academic/Plenum Books; 2001. pp. 83-101.

62. Guimón J, Zac S, eds. Challenges of psychoanalysis in the 21st century. New York: Kluwer Academic/Plenum Books; 2000.

63. Guimón J, Dávila R, Andreoli A, eds. Crisis y contención: del estrés a la estabilización terapéutica. Madrid: Eneida; 2007.

64. Guimón J, Totorica K, Villasana A, Ozamis A. Estudio comparativo de las opiniones sobre los contenidos psicológicos y psiquiátricos que se deben incluir en el 'curriculum' de la licenciatura en medicina. Actas Luso Esp Neurol Psiquiatr Cienc Afin 1980; 8: 179-90.

65. Salinsky J. The Balint movement worldwide: present state and future outlook: a brief history of Balint around the world. Am J Psychoanal 2002; 62: 327-35.

66. Stein HF. Reframing Balint: thoughts on family medicine departmental Balint groups. Fam Med 2003; 35: 289-90.

67. Tizón JL. Investigando sobre 'grupos Balint' o 'Grupos de reflexión’: ¿una técnica tan sólo docente? Ponencia Presentada en la Mesa Redonda sobre la Relación MédicoPaciente del 37 Congreso de la Sociedad Española de Medicina Psicosomática y Psicoterapia: La Psiquiatría de Enlace en el Siglo XXI. Madrid, España, abril de 2002.

68. Albaigés L, Castell E, Closas D, Dunyó F, Fernández E. El seminario 'Aprender a mirar de nuevo para poder transformar: observación directa'. Aportaciones a la formación del médico de familia. Aten Primaria 2007; 39: 437-42. 